\title{
METAMORFOSI DELLA SCRITTURA
}

Patrizia Calefato*

ABSTRACT: II concetto di "scrittura" fa riferimento non solo alla trascrizione alfabetica, ma all'intera attività umana di "inscrivere" il mondo con segni. Parole, immagini, numeri, racconti, testi: la scrittura è fatta di una materia composita che tocca tutti i nostri sensi. Oggi più che mai, la letteratura e le immagini, le parole e I corpi, le forme tradizionali della scrittura e le più avanzate tecnologie testuali si combinano tra loro quali espressioni della "scrittura" nel senso filosofico più profondo.

PAROLE CHIAVE: Scrittura. Lettura. Linguaggio. ICT. Metamorfosi.

Nella nostra epoca la scrittura mostra la sua complessità anche nella vita quotidiana. Malgrado il pregiudizio di chi asseriva che, con l'avvento delle nuove tecnologie della comunicazione e dell'informazione, l'umanità avrebbe perso la sua capacità di "usare" le parole, noi abbiamo oggi molte più possibilità di scrivere, e conseguentemente di leggere, rispetto a ieri.

Questo testo si concentra sulle metamorfosi che la scrittura sta vivendo nel nostro tempo. Spiegherò come concetti quali creatività, scrittura, lettura, nominazione, sono cambiati nell'era digitale e come le ICT stanno mostrando la complessità della scrittura. Per fare ciò, seguirò la guida ideale delle "Lezioni americane" (1985) dello scrittore italiano Italo Calvino. Sebbene egli sia morto nel 1985, quindi appena prima della rivoluzione informatica e digitale di fine Novecento, intuì molto bene i valori e le regole che la scrittura avrebbe dovuto preservare nel futuro. Le sue "raccomandazioni" riguardano la leggerezza, la rapidità, l'esattezza, la

\footnotetext{
* Dipartimento di Lettere Lingue e Arti: Italianistica e Culture comparate, Università degli studi di Bari Aldo Moro, Bari, Italia e Grupo de Investigación Hermeneia Estudios Literarios y Tecnologías Digitales, Universiat de Barcelona. E-mail: patrizia.calefato@uniba.it.
}

\section{(cc) EY-NC-ND}

Esta obra está licenciada com uma licença Creative Commons.

Texto Digital, Florianópolis, Santa Catarina, Brasil, v. 11, n. 2, p. 148-170, jul./dez. 2015. ISSNe: 1807-9288. 
visibilità e la molteplicità: parole chiave che paragonerò con la loro possibile realizzazione nell'era delle connessioni planetarie.

\section{Le metamorfosi della scrittura nell'era tecnologica}

Nella nostra epoca il segno scritto e la parola orale, la letteratura e le immagini, le parole e i corpi, le modalità più tradizionali della scrittura e della lettura e le forme tecnologicamente più avanzate della testualità, si contaminano reciprocamente e si presentano come svariate e molteplici manifestazioni della "scrittura" intesa nel senso più profondo e filosofico che questa parola contiene. "Scrittura", infatti, è un concetto che copre non solamente l'ambito della trascrizione alfabetica, ma l'intera attività umana dell'"incidere il mondo" con i segni. Parole, suoni, immagini, numeri, racconti, testi: la materia di cui la scrittura è fatta è composita, investe in modo plurimo i nostri sensi.

È particolarmente nell'epoca presente che questa complessità e pluralità del concetto di scrittura si rende tangibile ed esperibile anche nella vita quotidiana. A dispetto di quanti avevano sostenuto che con l'avvento delle nuove tecnologie avremmo perso quella capacità di "maneggiare le parole" che la scrittura con la penna, intesa in senso stretto, rende letteralmente possibile, abbiamo oggi a che fare con una possibilità di scrivere (e conseguentemente di leggere) moltiplicata quantitativamente e qualitativamente. La mano non guida più solo la penna per tracciare dei segni sulla carta, ma batte sulla tastiera, guida il mouse, sfiora il touch-pad, tocca coi polpastrelli lo schermo di un tablet per selezionare, allargare, sfogliare pagine elettroniche, compie gesti elementari come quello di picchiettare con il plettro sul display di uno smartphone. Alla mano si accompagna la voce, che può comandare le funzioni di un computer; si accompagna la vista, attraverso cui "leggiamo" non solo sequenze alfabetiche, ma anche e in modo crescente sequenze visive, in quel "cinema mentale" di cui parlava Italo Calvino, in cui consiste la basilare capacità immaginativa del pensiero come linguaggio.

Calvino: lo scrittore italiano (1923-1985) intuì in modo speciale i valori che la scrittura avrebbe dovuto preservare nel millennio presente. Le sue Lezioni Texto Digital, Florianópolis, Santa Catarina, Brasil, v. 11, n. 2, p. 148-170, jul./dez. 2015. ISSNe: 1807-9288. 
americane (1985) parlavano di leggerezza, rapidità, esattezza, visibilità, molteplicità: parole chiave che si potrebbero oggi mettere a confronto con le loro possibili realizzazioni rese realtà dalla civiltà delle tecnologie e delle connessioni planetarie.

La leggerezza appartiene al "potere leggero del software" di cui già lo scrittore ben individuava la portata epistemologica.

La rapidità concerne - come dice Calvino richiamandosi in questo a Leopardi - il rapporto tra la velocità mentale e quella fisica (CALVINO, 1985, p. 665) che evoca l'idea di infinito e che costantemente mettiamo alla prova quando valutiamo quali effetti produca sul nostro quotidiano "lavoro segnico" la velocità di un processore; quando verifichiamo la velocità di funzionamento di un motore di ricerca; quando esigiamo velocità nello scambio comunicativo in una chat o nella posta elettronica.

L'esattezza riguarda invece per Calvino la precisione, la nitidezza, la definizione dei concetti: una prerogativa che oggi fa i conti con il mare di informazioni in cui siamo immersi che ci pone direttamente l'esigenza da un lato di trasparenza, dall'altro di selezione dei significati: il mare di file che WikiLeaks ha reso disponibili in rete nel dicembre 2010 e che hanno messo in difficoltà i rapporti tra le potenze mondiali, ad esempio, esprime proprio questa duplice esigenza.

La visibilità si conferma a pieno come quel "mondo, o golfo mai saturabile di forme e di immagini" (CALVINO, 1985, p. 706), che potrebbe oggi essere il mondo dei blog e dei social network.

La molteplicità ha a che vedere infine con le connessioni, l'enciclopedia del sapere, le reti "tra i fatti, tra le persone, tra le cose del mondo" (CALVINO, 1985, p. 717): un'immagine che rende efficacemente, senza certo che lo scrittore italiano lo sapesse, i tratti essenziali del web.

La "società dell'informazione" oggi realizzata non ha certo in sé quegli aspetti salvifici che alcuni forse si aspettavano di poter raggiungere solo con il supporto Texto Digital, Florianópolis, Santa Catarina, Brasil, v. 11, n. 2, p. 148-170, jul./dez. 2015. ISSNe: 1807-9288. 
della tecnologia. La dimensione planetaria della comunicazione e I'intelligenza" delle macchine della comunicazione non sono infatti sufficienti di per sé a garantire il rigenerarsi virtuoso e più alto di parole come democrazia, alfabetizzazione, lavoro, trasparenza, diritti, conoscenza. La scrittura può essere allora un elemento chiave per impegnare le tecnologie in progettazioni sociali in cui tali parole siano invece affermate nel loro senso più ricco e più universale. La scrittura, infatti, è contemporaneamente la precondizione della produzione di segni e il modo in cui i segni riescono a collegarsi all'esperienza umana più autentica.

\title{
L'insostenibile leggerezza dei bit
}

Tra le citazioni e gli esempi letterari, mitologici e culturali che Calvino utilizza per parlare della leggerezza, c'è un passaggio che fa riferimento all'informatica. Scrive Calvino:

\begin{abstract}
Poi l'informatica. Ė vero che il software non potrebbe esercitare i poteri della sua leggerezza se non mediante la pesantezza del hardware; ma è il software che comanda, che agisce sul mondo esterno e sulle macchine, le quali esistono solo in funzione del software, si evolvono in modo d'elaborare programmi sempre più complessi. La seconda rivoluzione industriale non si presenta come la prima con immagini schiaccianti quali presse di laminatoi o colate d'acciaio, ma come i bits d'un flusso d'informazione che corre sui circuiti sotto forma d'impulsi elettronici. Le macchine di ferro ci sono sempre, ma obbediscono ai bits senza peso (CALVINO, 1985, p. 636).
\end{abstract}

Nel 1985, anno in cui Calvino scriveva queste righe, anno anche della sua morte che sarebbe avvenuta in settembre, il ruolo che nel giro di poco tempo l'informatica avrebbe svolto nella scrittura, nelle pratiche di linguaggio e nella vita quotidiana poteva essere solo intuito. Come scrittore, Calvino guardava le cose certamente da un punto di vista privilegiato: più acuta di altre prospettive sul mondo è infatti quella che la letteratura apre, permettendo allo sguardo dello scrittore e della scrittrice di non fermarsi al qui ed ora, alla banale e piatta attualità, bensì di vedere, e pre-vedere in certi casi, le trasformazioni significative della società, illuminati da una singolare saggezza. 
II "potere leggero" del software, di cui così efficacemente Calvino ci offriva l'immagine, ha caratterizzato effettivamente la rivoluzione informatica inauguratasi alla fine del XX secolo e si è insediato ormai stabilmente. I bit senza peso comandano sugli atomi ponderosi; i segni dominano sui corpi. Parlo qui della ripartizione semiotica tra segni e corpi, parallela a quella tra bit e atomi nella quale il bit è l'unità elementare dell'informazione digitalizzata, mentre l'atomo ne è la componente misurabile, fisica (CALEFATO, 2007, pp. 121-125; NEGROPONTE, 1995). Nella citazione sopra riportata, Calvino utilizza l'espressione "bit" anche in senso metaforico per indicare la parte informativa, segnica, che si impone, programmandola, sulla parte "pesante" degli atomi di cui sono composte le macchine. Analogo rapporto vi è tra il segno, in quanto elemento leggero, aperto a più percorsi dell'interpretazione, e il corpo quale residuo non interpretabile del segno, elemento anch'esso, come l'atomo, pesante, esteso, tangibile.

Ad esempio, un tablet o un iPad è dotato di un suo "corpo", nel senso che è fatto di certi materiali, ha una forma, una consistenza, un colore. L'iPad, come tutti $\mathrm{i}$ media, ha però fondamentalmente una funzione segnica, comunicativa: in esso sono i "bit" a prevalere sugli "atomi", i segni a prevalere sul corpo. Non dimentichiamo però quanta importanza abbia oggi l'aspetto estetico anche del mezzo di comunicazione: dal televisore del salotto di casa al telefono cellulare, all'iPad appunto, che nel vicino futuro sarà probabilmente uno degli oggetti ricordati tra i simboli del nostro decennio, come il Walkman a cassette lo fu per gli anni ' 80 o il registratore a nastri per gli anni '60.

La società dell'informazione è la società dei bit, dei segni, della leggerezza. Si tratta di una leggerezza non sempre però inquadrabile nell'immagine positiva di cui ci parla Calvino. Tutto ciò che oggi possiamo estesamente o metaforicamente comprendere nella nozione di bit, infatti, cioè i software e le applicazioni che fanno funzionare computer, robot, macchine, telefoni cellulari, palmari, netbook, smartphone, iPod, iPad, e ogni altro oggetto definibile anch'esso estesamente e metaforicamente come hardware, sono allo stesso tempo sia il motore che il risultato di un modo di produzione preciso, che è quello del capitalismo informatizzato. In tale processo assumono un ruolo determinante il lavoro Texto Digital, Florianópolis, Santa Catarina, Brasil, v. 11, n. 2, p. 148-170, jul./dez. 2015. ISSNe: 1807-9288. 
intellettuale umano, il sapere, la conoscenza, che molto spesso però, invece che venire valorizzati e resi protagonisti del processo di produzione, vengono a trovarsi nella stessa condizione di quel lavoro astratto, generale, dal quale il capitalismo della prima rivoluzione industriale traeva la sua forza produttiva principale in grado di erogare plusvalore. È sulla riduzione alienante e sull'umiliazione di questo genere di lavoro segnico, linguistico, cosiddetto "immateriale" (ma quanta materialità vi è nel sapere, nei segni, nei bit!), che si fonda la moderna ideologia neoliberistica della "leggerezza". Essa ha fatto ormai della provvisorietà, della precarietà, dell'incertezza, i nuovi perni del lavoro e dell'esistenza di milioni di persone. Invece di essere un valore, questo genere di leggerezza diviene così una dannazione.

Il richiamo a Calvino è allora fondamentale, in quanto la concezione che egli ha di questo valore da salvare per il millennio in cui noi siamo, al quale egli guardava con preoccupazione, contiene la chiave di una critica culturale e può forse rappresentare il puntello per transvalutare la leggerezza "mordi e fuggi" dell'ultraliberismo in qualcosa che ci invita a pensare e agire liberamente e criticamente. Un invito che muove a partire dalla consapevolezza che leggerezza non è sinonimo di frivolezza:

[...] spero innanzi tutto d'aver dimostrato che esiste una leggerezza della pensosità, così come tutti sappiamo che esiste una leggerezza della frivolezza; anzi, la leggerezza pensosa può far apparire la frivolezza come pesante e opaca (CALVINO, 1985, p. 638).

La leggerezza non è neanche superficialità. Partendo dall'immagine di Guido Cavalcanti così come ci viene presentato da Boccaccio nel Decameron nell'atto di spiccare un salto che gli permette di sfuggire ai buontemponi fiorentini che volevano "dargli briga", Calvino ci mostra infatti come simbolo augurale per il terzo millennio

l'agile salto del poeta-filosofo che si solleva sulla pesantezza del mondo, dimostrando che la sua gravità contiene il segreto della leggerezza, mentre quella che molti credono essere la vitalità dei tempi, rumorosa, 
aggressiva, scalpitante e rombante, appartiene al regno della morte, come un cimitero d'automobili arrugginite (CALVINO, 1985, p. 639).

È un'immagine di foresta pietrificata, quella del mondo odierno colto in un processo di trasformazione, che in più passaggi dei suoi scritti Calvino paventava e verso cui suggeriva strategie di resistenza affidate soprattutto al linguaggio. Purché la parola sia in grado di essere "inseguimento perpetuo delle cose, adeguamento alla loro varietà infinita" (CALVINO, 1985, p. 653). Purché la foresta pietrificata sia guardata come fa Perseo con la Medusa, che, volando su sandali alati, non rivolge mai alla Gorgone lo sguardo diretto, "ma solo sulla sua immagine riflessa nello scudo di bronzo" (CALVINO, 1985, p. 632). È la scrittura a permettere questo sguardo indiretto sulla Gorgone-mondo, quella scrittura i cui confini si sono estesi a dismisura, nel mondo dei bit e dei software imperanti, quella scrittura che però richiede di essere richiamata alla sua "intransitività" (BARTHES, 2002, p. 13-22; PONZIO 2006, pp. 21-24).

A dispetto delle apocalittiche considerazioni sulla presunta fine della scrittura, e della connessa fine della lettura, che avrebbero accompagnato la rivoluzione informatica del nostro tempo, il mondo attuale è pervaso di pratiche di scrittura e di lettura legate proprio ai nuovi media. Gran parte di quel lavoro segnico di cui parlavo poc'anzi si fonda sulla parola scritta, gran parte del tempo di vita è oggi un tempo dedicato alla scrittura e alla lettura, dalle e-mail agli sms, dai blog ai social network, dalle geolocalizzazioni al turismo online. Certo, non si tratta, se non per una piccolissima percentuale di casi, di scritture e letture impegnative o "colte": un sms non è un poema, un blog non è un romanzo, una schermata su una qualsiasi homepage non è la visione immaginifica e fantastica che può offrirci una pagina di Shakespeare o di Ariosto. II più delle volte si tratta in effetti di scritture e letture che Roland Barthes avrebbe chiamato "transitive", cioè finalizzate a trasmettere un messaggio e non a valere in sé come testi che non hanno alcun compito, né di informazione, né di verità, né di testimonianza. La "transitività" è infatti propria degli "scriventi" contrapposti agli "scrittori", figure che Barthes così definisce: 
[...] lo scrittore è il solo, per definizione, a perdere la propria struttura e quella del mondo nella struttura della parola (BARTHES, 2002, p. 141).

[...] Gli scriventi, invece, sono uomini "transitivi"; si pongono un fine (testimoniare, spiegare, insegnare) di cui la parola non è che il mezzo (BARTHES, 2002, p. 143).

Ciò di fronte a cui ci mette la "scrittura elettronica" di oggi è però una ridiscussione di questa dicotomia che Barthes individuava in modo netto. Quanta "intransitività" è per esempio contenuta in quegli sms che ci si scambia tra amici o tra partner solo per il gusto di mantenere vivo il rapporto a distanza, poca o tanta che sia; quanto invischiati nella "struttura della parola" siamo nel momento in cui produciamo e consumiamo parole nel web, fossero anche i "tweet" o i "cambiamenti di status" su un social network? Poca, certo, dal punto di vista della riproduzione sociale, dal momento che ogni sms è rigorosamente quantificato in centesimi consumati sulla scheda telefonica $O$ in superofferte estive ricambiate però da onerose ricariche. E ogni status su Facebook viene immagazzinato nella memoria sociale di "mi piace" e stili di vita di cui è poi il marketing ad alimentarsi.

Molta intransitività, di contro, può essere intesa dal punto di vista dell'esercizio della scrittura in cui la nostra epoca si è nuovamente avventurata: una scrittura pur "illetterata" e banale, priva di senso del tempo e della storia, come dimostrano i test universitari, spesso disastrosi nei risultati riguardanti la conoscenza della lingua standard. E malgrado l'analfabetismo di ritorno di adulti colti che, a forza di cimentarsi con acronimi, emoticone e velocità degli enunciati, dimenticano l'ortografia, la sintassi e l'uso della punteggiatura. Nonostante questi aspetti qui sintetizzati forse in modo troppo perentorio, non v'è dubbio che siamo decisamente una società dedicata alle attività della scrittura e della lettura, soprattutto di forme brevi, certo, ma sempre di scrittura e lettura si tratta: dagli sms ai tweet già citati; dai fastidiosissimi titoli di news che scorrono sotto i mezzibusti dei moderni telegiornali agli slogan e marchi pubblicitari che tappezzano le nostre città; dai testi che appaiono sulle T-shirt ai logo incisi sugli indumenti e gli accessori. 
Qual è il confine allora oggi tra scrittori e scriventi, per seguire le definizioni di Barthes, o tra lettori e leggenti? Non contiene in fondo sempre la pratica della scrittura un'aspirazione che va al di là del messaggio e al di là della funzionalità? A maggior ragione quando la scrittura è parte integrante del mezzo che la rende possibile; quando la scrittura si mostra nel momento stesso in cui si forma sotto le dita sulla tastiera, sotto lo sguardo sul monitor o sotto il contatto tra le mani e il touch-screen. Tutti, nella scrittura elettronica, perdono un po' "la propria struttura e quella del mondo nella struttura della parola", per usare l'espressione attraverso cui Barthes definisce lo scrittore; anche se questa struttura della parola è ridotta all'osso, anche quando non è un problema di stile o di metafore, anche quando la scrittura non crea universi letterari, ma si limita a raffigurare la sua stessa scena.

\section{La scena della scrittura}

In Della grammatologia il filosofo Jacques Derrida critica l'antropologo Claude Lévi-Strauss per la sua definizione dei Nambikwara come popolo "privo di scrittura". Dire che un popolo "non sa scrivere", dice Derrida, equivale a rifiutargli la parola oppure a tradurre la parola di questo popolo con un "gridare", un "cantare", un "soffiare", e soprattutto con il "balbettare" (DERRIDA, 1988, p. 103). Balbettare è una parola onomatopeica che viene dal greco "barbaros", quest'ultima onomatopeica anch'essa dato che riproduce nel suo significante il "suono" confuso e allitterato che i greci presumevano fosse la lingua degli stranieri. Anche in latino abbiamo un'analoga onomatopea con il termine "balbus" che vuol dire proprio "balbettio". Quando diciamo "barbari", sia per definire quelli che per i Greci o i Romani erano gli stranieri, sia, nel nostro tempo, per valorizzare negativamente popoli "altri" ed usanze come per esempio il velo islamico o alcuni decenni fa il rogo delle vedove in India, o anche pratiche oggi di moda come il piercing, stiamo usando una parola che inscrive in sé la stereotipizzazione della lingua dell'altro. $E$ non è una semplice questione di etimologia, o meglio l'etimologia spesso racconta il senso e il valore sociale comune depositato nelle parole. 
L' "essere senza scrittura" dei barbari si pone come contraltare del pregiudizio etnocentrico, logocentrico, della scrittura come "fare righe" (nell'espressione di Derrida), cioè come trascrizione, dignità e forza di legge che la parola assumerebbe nella sua permanenza (diciamo infatti scripta manent). La "scrittura" che ci ha insegnato invece a concepire Derrida ha a che vedere non con la sua permanenza, ma pone invece quali valori: la traccia, il tempo inteso come spaziatura e ritmo, il distanziamento, la différance (termine da lui coniato), come testimonianza di uno scarto non colmabile, differimento che è anche nel tempo e dal tempo, è un debordare rispetto a categorie generalizzanti e universali.

Questa scrittura è incisione e stilo, "sprone", parola su cui ritornerò tra breve. Questa scrittura pone le basi per quello che mi piace chiamare lo "spartito teorico" in cui suona la filosofia di Derrida: la decostruzione. "Decostruzione" è un termine che viene dal lessico filosofico heideggeriano poiché quando compare le prime volte in Derrida è "distruzione", una parola che doveva essere inclusa nel titolo della seconda parte dello scritto di Heidegger Kant e il problema della metafisica. Ma la decostruzione - che certo qui non può essere esaurita in brevi e povere parole - , è una pratica teorica, come la definisce Gayatri Spivak, traduttrice americana di Della Grammatologia nel 1976, e dico "traduttrice", nel senso grande e stratificato che questa parola e il compito del traduttore hanno. Una pratica teorica che mette in moto una tra svalutazione, per usare un termine nietzscheano, permanente, una apertura, uno squarcio, una incisione costante con lo stilo, una "scrittura" fendente nella testualità e nei segni, una traccia.

"Traccia" si dice in tedesco Spur. La stessa parola "spur" in inglese vuol dire "sprone", éperon in francese. Tra queste quattro lingue, gioca Derrida in un suo scritto bellissimo del 1976, pubblicato in prima raffinata edizione a Venezia e intitolato Eperons (Sproni), su "gli stili di Nietzsche". È uno scritto in cui la decostruzione mostra all'opera i suoi fendenti, i suoi sproni, le Spuren, le tracce, incise nello stile-stilo della scrittura di Nietzsche. Scrive Derrida: 
ostinatamente: la presenza, dunque, il contenuto, la cosa stessa, la verità - a meno che, in tutto questo disvelarsi della differenza, non si tratti già di abisso deflorato (DERRIDA, 1976, p. 33-34).

È la presenza, la verità coi suoi "fattori", intesi come coloro che la producono (da facio) e come coloro che la "portano" (da fero), che la decostruzione apre e sprona, mettendosi all'opera proprio a partire da dove si costruisce il suo fondamento velato, la sua origine "sotto cancellatura".

Nel Fedro, Platone racconta, attraverso la voce di Socrate, il mito del dio egizio Theuth, inventore dei numeri, del calcolo, della geometria, dell'astronomia, del gioco del tavoliere e dei dadi e delle lettere dell'alfabeto (PLATONE, Fedro, LIX, 274, c-d). Con quest'ultima invenzione, Theuth diede agli egiziani il dono della scrittura alfabetica, dono che in verità Socrate non apprezza particolarmente, poiché considera la scrittura né più né meno che un supporto strumentale della memoria. Alla scrittura, Socrate antepone invece il discorso parlato come viva dialettica nel linguaggio e testimonianza di autentica sapienza,delle quali il discorso scritto può solo presentarsi come immagine (PLATONE, Fedro, LX, 276, a).

Come tutti i dialoghi platonici, anche il Fedro permette di cogliere delle suggestioni che attraverso il mito aprono ad accostamenti e interpretazioni possibili, non riducibili a un unico percorso. Una di queste interpretazioni può essere quella secondo cui, al di là delle perplessità di Socrate sulla scrittura, ci si soffermi sull'insieme dei doni di Theuth. L'immagine del dono della scrittura alfabetica fatto da Theuth insieme a quello gioco, della matematica, dell'astronomia e della geometria, ci permette così di ipotizzare una "scena della scrittura" in cui convivono alfabeto e numeri, forme astratte e forme dell'universo. In questa "scena", ciò che conta non è di per sé quanto è scritto, ma le forme in cui la scrittura si pone in relazione con gli altri doni del dio.

Gli antropologi della scrittura ci dimostrano come essa sia il modo attraverso cui il corpo si inscrive nel mondo, entra in contatto con questo. La scrittura dà ritmo, misura, forma, senso a tale contatto. Vi è nella scrittura una funzione di Texto Digital, Florianópolis, Santa Catarina, Brasil, v. 11, n. 2, p. 148-170, jul./dez. 2015. ISSNe: 1807-9288. 
"modellizzazione primaria del pensiero", come ha scritto Giorgio Raimondo Cardona (CARDONA, 1981, p. 51), che mette in discussione quella funzione mnemotecnica che Socrate biasimava, perché dimostra come le elaborazioni mentali abbiano bisogno, nel loro stesso costituirsi, di una semiotica che le strutturi, sia pur solo provvisoriamente.

C'è però ancora dell'altro da scavare nel mito: Theuth è un dio egizio, possiamo quindi immaginare che l'alfabeto che egli regala al suo popolo andrà a sostituire i caratteri geroglifici che appartenevano alla sua cultura più antica e la cui natura, come quella dei segni pittografici in genere, non è convenzionale e arbitraria, come nel caso del segno alfabetico, bensì analogica e pittorica. Tuttavia anche l'origine del segno scritto alfabetico è legata ai diversi modi in cui sono stati simbolizzati, nella storia delle culture, le tracce che il corpo lascia dietro di sé e il rapporto tra il corpo e il mondo circostante. Pensiamo solo al fatto che all'antropologia della scrittura siano state accostate pratiche significanti come quella del tessere, da cui la parola "testo", e del cantare. Persino l'alfabeto richiama allora alla luce il fatto che la scrittura sia qualcosa che, allo stesso tempo, dà senso alla lingua che trascrive e ha senso di per sé, anche a prescindere dai contenuti che trasmette.

Il carattere della scrittura è un carattere discreto, non continuo, perché i segni scritti sono entità isolate, che - come abbiamo detto - valgono di per sé, sebbene prendano senso nel loro comporsi combinatorio. Questo carattere discreto accomuna la scrittura a un'altra pratica: quella del contare, del numero. La scrittura sumera di Uruk (IV millennio a.C.), una delle più antiche scritture conosciute, registrava proprio liste, conti, inventari (SANGA, 2009, p. 41). Succede allora che le stesse caratteristiche che la funzione contabile della scrittura aveva per i Sumeri possano essere considerate le caratteristiche fondamentali della scrittura in generale, ulteriori elementi di quella scena in cui la scrittura è "discreta, combinatoria, lineare, analitica, astratta" (SANGA, 2009, p. 46). Tornano, in queste immagini, a ricomporsi nel loro reciproco rapporto i doni di Theuth e le attività umane connesse a questi doni: scrivere, contare, dare ritmo e senso al tempo, inscrivere il corpo nell'universo, tessere, cantare, mettere alla Texto Digital, Florianópolis, Santa Catarina, Brasil, v. 11, n. 2, p. 148-170, jul./dez. 2015. ISSNe: 1807-9288. 
prova il caso nel gioco. La scrittura è allo stesso tempo lavoro (tessere) e attività infunzionale (gioco, canto); misura (numero, ritmo) e apertura all'imprevedibile (gioco).

Come si riproduce la scena della scrittura nel contesto delle tecnologie informatiche e digitali del nostro tempo?

\section{Informatica e linguistica}

In un saggio degli anni '90 che analizzava i rapporti tra linguistica e informatica, Tullio de Mauro presentava "una mappa di intersezioni possibili" tra queste due discipline la cui comune materia di riferimento è il linguaggio. Le intersezioni proposte da De Mauro erano le seguenti:

1. L'informatica come oggetto di studio sociolinguistico e storico-lessicologico;

2. L'informatica come complemento di indagini linguistiche descrittive;

3. L'informatica come strumento dell'attività linguistica dei parlanti e degli scriventi;

4. L'informatica come fonte di stimoli critici per la linguistica teorica.

Tra queste, due mi paiono qui di particolare interesse in quanto richiamano direttamente in causa il ruolo della scrittura e aprono delle risposte alla domanda posta alla fine del precedente paragrafo. La prima fa riferimento al punto 3 e si esplicita in quella che De Mauro chiama "l'attività di autocontrollo" dell'attività linguistica di parlanti e scriventi; la seconda fa riferimento al punto 4 e riguarda strettamente il rapporto tra la lingua e il calcolo numerico. Cominciamo dal primo aspetto: esiste, dice De Mauro, una "componente riflessa" (DE MAURO, 1999, p. 112) caratteristica del linguaggio verbale che ci permette, ad esempio, di correggere da soli nel parlato i frequenti errori, di ordine sia lessicale che semantico e sintattico, che commettiamo. Questo autocontrollo del linguaggio non si motiva tanto in una ricerca della correttezza a tutti i costi, bensì nella necessità di porci dal punto di vista di chi interagisce con noi nella comunicazione, che ha bisogno di comprendere quanto diciamo. Nell'oralità moduliamo costantemente questa componente: chi più chi meno, certo, e a condizione che chi parla, per esempio in una conferenza o una lezione, guardi sempre negli occhi chi ascolta in Texto Digital, Florianópolis, Santa Catarina, Brasil, v. 11, n. 2, p. 148-170, jul./dez. 2015. ISSNe: 1807-9288. 
modo da cogliere ogni implicita domanda di chiarimento, ogni incomprensione, ogni calo dell'attenzione. E non è semplice.

Ancora più complessa tale autocorrezione risulta nello scritto, dove, dice De Mauro con riferimento a Italo Calvino, un bravo scrittore - in questo caso non necessariamente scrittore di professione - "deve sempre essere pronto a questa operazione di spiazzamento, a saltare, nello scrivere, dalla parte dei suoi desiderati lettori” (DE MAURO, 1999, p. 113). L'informatica è oggi in grado di aiutare la scrittura in questa operazione di "spiazzamento", permette di saltare dalla parte di chi scrive alla parte di chi legge, dando, per così dire, leggerezza alla parola, proprio nel senso di permetterle balzi simili a quello che compie Guido Cavalcanti nel Decameron. Le scritture elettroniche sono dotate infatti di meccanismi di autocorrezione, dal semplice correttore ortografico di Word ai sistemi che il web ci offre per definire meglio termini e concetti attraverso i motori di ricerca, per utilizzare sinonimi, per mettere a confronto tra lingue diverse parole e testi, perfino per realizzare traduzioni.

Proprio quest'ultimo tema, quello della traduzione, offre però a De Mauro l'occasione di una domanda cruciale: "la lingua è un calcolo?" (DE MAURO 1999, p. 115). E qui passiamo alla seconda intersezione tra linguistica e informatica, quella cioè relativa al rapporto tra il linguaggio verbale e il computo matematico, dove il caso della traduzione si mostra come esemplare. L'esperienza di decenni di tentativi ha dimostrato infatti come sia impossibile una traduzione totalmente automatizzata. La traduzione è infatti una forma di testualità talmente particolare, talmente aperta e "leggera", ma non per questo inconsistente, che solo i testi molto formalizzati, chiusi e delimitati, possono venir tradotti automaticamente, e anch'essi sempre con una certa approssimazione che rende ogni volta necessario il controllo "umano" su quello dell'”automa". Come scrive De Mauro,

È certa la possibilità di assistenza informatica alla traduzione di testi di particolare natura, limitati a determinati ambiti di contenuto e con un lessico e una sintassi ben delimitati e chiusi, insomma di testi costruiti in una lingua e ricostruibili in un'altra in obbedienza a un certo numero (finito) di regole aggiuntive a quelle usate comunemente dai parlanti, testi dunque in certa misura formalizzati (DE MAURO 1999, p. 114).

Texto Digital, Florianópolis, Santa Catarina, Brasil, v. 11, n. 2, p. 148-170, jul./dez. 2015. ISSNe: 1807-9288. 
Ma è "il senso particolare di un testo o anche di una sola frase di un testo in una lingua storico-naturale" (DE MAURO, 1999, p. 115) a non potere essere né inteso nella lingua di partenza, né reso in un'altra lingua nemmeno dal più complesso elaboratore. Su questo non ha dubbi De Mauro, che lascia comunque aperta la possibilità di poter applicare il calcolo a una parte degli usi delle lingue naturali, in quanto la lingua è, scrive, "un insieme sufficientemente plastico" per potere ammettere tali applicazioni.

Le pratiche di analisi linguistica dei corpora sono un esempio di questa prerogativa che le lingue offrono. Fondata su base lessicologica e avviatasi sin dai primi anni '60, la linguistica dei corpora utilizza oggi dei software per estrapolare da un testo - sia scritto che orale - il maggior numero di informazioni possibili relative fondamentalmente alle occorrenze di parole, espressioni, frasi. Molto utili come supporto per lo studio delle lingue straniere, per la traduzione, per la conoscenza storica delle lingue naturali, i corpora non sono però in grado di esaurire l'analisi di un testo, né di comprendere in sé la complessità della pratica della scrittura.

Come scrive Italo Calvino, "le lingue naturali dicono sempre qualcosa in più rispetto ai linguaggi formalizzati (CALVINO, 1985, p. 691)". Malgrado sia indubitabilmente vero quanto dice De Mauro, cioè che

numerazione, calcolo aritmetico, assiomatizzazioni delle matematiche e di altre scienze dure, determinazioni di regole d'uso dei termini delle scienze e tecniche hanno la loro radice e atmosfera vitale dentro le lingue storico-naturali (DE MAURO, 1999, p. 116),

è comunque vero che il linguaggio e la scrittura non sono semplice calcolo, né nomenclatura, né inventario di lemmi, ma sono esperienze, pratiche sociali, s' inverano in discorsi e in generi discorsuali: consistono insomma nel complesso lavoro segnico di individui in carne e ossa.

\section{Creatività e scrittura}


Una buona parte di questo lavoro segnico coinvolge oggi chi si "imbarchi", per necessità o per gusto, nella scrittura di testi definibili di genere "saggistico" in vari ambiti, ad esempio articoli di tipo giornalistico, saggi di ricerca, saggi brevi, temi, recensioni, tesi di laurea, tesine d'esame. Aggiungo a queste forme di testualità anche altre pratiche di scrittura: quelle che attualmente vengono definite "note" nel contesto di un social network come Facebook, i post dei blog, i "commenti" negli stessi ambiti, e mi arrischio a introdurre in questo novero anche testi brevi come quelli che esprimono gli "stati" in luoghi del web, come lo stesso Facebook, come Whatsapp, o come Twitter, e quanto ancora potrà venire, nel tempo prossimo, a coniugare l'esigenza perenne di scrittura che caratterizza gli esseri umani con la ugualmente perenne mutevolezza delle tecnologie della comunicazione. Aggiungo ancora un genere particolare di scrittura, quella epistolare propria delle e-mail.

Non possiamo escludere dall'ambito delle scritture in metamorfosi di cui questo libro tratta alcuni tipi di scrittura che Barthes chiamerebbe "funzionale", quella cioè finalizzata a veicolare messaggi direttamente spendibili nel mercato in senso stretto: dagli slogan pubblicitari, ai marchi, ai concept aziendali, ai testi del marketing, ai discorsi scritti per essere letti in pubblico e a tutto ciò che riguarda la scrittura fatta per "la comunicazione". II modello classico cui si fa riferimento quando si parla di "comunicazione" è quello che Roman Jakobson (JAKOBSON, 1963) ha espresso grosso modo nei seguenti termini: un messaggio viene scambiato tra un emittente che produce il messaggio e un ricevente che riesce a decodificarlo in virtù di un codice di riferimento comune condiviso con l'emittente. Questo modello molto schematico non tiene conto però della complessità che il concetto di comunicazione ha assunto soprattutto nella nostra epoca. II messaggio non è infatti un "pacco postale", come diceva ironicamente Rossi-Landi (ROSSILANDI, 1980, p. 207) che passa di mano in mano senza nello stesso tempo modificarsi, o esporsi al rischio di fraintendimenti, o ancora arricchirsi di nuovi significati, o viceversa perdersi in una entropia dovuta a troppa informazione circolante.

Come già accennato prima, riesce molto difficile oggi discernere tra scritture transitive e intransitive, tra scrittori e scriventi. A maggior ragione in un'epoca in Texto Digital, Florianópolis, Santa Catarina, Brasil, v. 11, n. 2, p. 148-170, jul./dez. 2015. ISSNe: 1807-9288. 
cui la "creatività" viene chiamata in causa proprio in settori come il marketing, la comunicazione pubblicitaria, quella politica e quella della moda. Cos'è la creatività, se non l'investimento passionale, corporeo, emozionale, caratteristico di quelle attività legate alla conoscenza, al sapere e alla comunicazione, cioè a quel lavoro segnico del quale la scrittura rappresenta un elemento fondamentale e modellizzante? Comunicazione allude a ciò che c'è di comune, vuol dire messaggi, informazioni, linguaggi, emozioni, saperi, conoscenze, corpi, generi sessuali, tempo, spazio. Vuol dire oggi una totalità del tempo di vita da cui non si distingue più il tempo di lavoro, perché il lavoro è ovunque - dalla catena di montaggio (che tuttora esiste, non è stata abolita in quello che impropriamente alcuni chiamano "lavoro immateriale"), agli sms del telefonino, allo shopping natalizio, alla posta elettronica.

Eppure si dice che "il lavoro non c'è". Ma è vero questo? O non c'è piuttosto cioè un lavoro e un valore diffusi, valore vivo, valore sociale, valore in quanto sapere, valore per me in quanto "lavoratrice segnica" intellettuale, per l'immigrato in quanto "valore in traduzione" poiché porta con sé una o più lingue, una literacy cioè un'alfabetizzazione e insieme una conoscenza culturale complessa - una cucina, un sapere sul corpo, un patrimonio letterario, una fede, un immaginario, dei simboli? È lavoro, valore e scrittura quello della scrittrice quotidiana di un blog che mette alla prova, da dilettante, conoscenze e saperi altamente elaborati: l'html, photoshop, il linguaggio poetico. È lavoro-valore-scrittura quello dell'artista urbano che spruzza graffiti sui muri; quello del dj o vj che campionando e mixando rilavora il suono e l'immagine; quello del gruppo di stagisti a costo zero in un ufficio pubblico o in un'azienda privata, che mettono in piedi un ufficio stampa con tutti i crismi e insegnano ai vecchi impiegati come si fa.

Singolare se non addirittura poco pertinente è dunque oggi definire i confini tra la scrittura "creativa" e quella "non creativa". Certo, io stessa, nell'elencare ambiti ed esempi di scritture, ho escluso volutamente l'ambito dei generi letterari poetici e narrativi. Chi voglia scrivere un romanzo, un racconto o un testo poetico ha effettivamente dei criteri precisi da seguire: raccontare una storia, costruire dei personaggi e dar loro dei nomi, immaginarsi un tempo e uno spazio di azione, nel Texto Digital, Florianópolis, Santa Catarina, Brasil, v. 11, n. 2, p. 148-170, jul./dez. 2015. ISSNe: 1807-9288. 
caso del romanzo e del racconto. Concepire un ritmo, un metro, far lavorare la metafora e liberare le parole, nel caso del testo poetico. Non è però possibile escludere la dimensione "poetica", "letteraria", o comunque definibile, come abbiamo poc'anzi detto, "creativa", dai generi testuali che le metamorfosi della scrittura hanno creato nel nostro tempo e che usiamo quotidianamente, per esempio quando creiamo un nickname.

\section{II corpo nella scrittura elettronica}

Il web è solcato da innumerevoli percorsi di senso, da voci, da corpi, da impulsi e da risa, come una piazza o una strada intasata di gente. È lì che ci possiamo isolare di notte in una stanza dalle pareti trasparenti per tirare a indovinare sullo sconosciuto che le attraverserà chiamandoci. È lì che si può far chiasso in tanti, un coro stonato di battute asincrone che si susseguono tra finestre aperte e diavolerie infantili di emoticone e squilli. È lì che si deposita sapere anonimo e senza etichetta, si scarica e si cerca, si pubblicano e si condividono immagini, musica, filmati, scrittura. Ė lì che inviamo e riceviamo messaggi di posta infinita, pronta, se solo volessimo, a rimanere inevasa per i prossimi diecimila anni. È lì che indossiamo i nostri nickname.

I nickname interpretano la vocazione umana a comporre letteratura. È sempre stata, questa, una prerogativa dei soprannomi: piccoli testi in grado di racchiudere in un solo segno il corpo e il riso, e molto spesso un riso che sa di derisione, di messa alla berlina. II nick è, come il soprannome, un segno cui si giunge con arte, ma che, a differenza di quello, è il "proprietario" stesso a conquistarsi come un piccolo trofeo. I ragazzi che chattano su MSN sono in grado di cambiarne uno ogni giorno, a seconda dello stato d'animo o della musica che ascoltano nella playlist del momento, e riescono sempre a ben accordarlo con un motto, un desiderio, una provocazione, che vengono depositate sul proprio "biglietto da visita". Un modo in cui concedersi o negarsi a chi ci "possiede", ma solo nella sua lista di contatti.

La tecnologia diffusa ci ha resi tutti più in grado di essere inventori di parole: quante password dobbiamo coniare e ricordarci ogni giorno per accedere ai server Texto Digital, Florianópolis, Santa Catarina, Brasil, v. 11, n. 2, p. 148-170, jul./dez. 2015. ISSNe: 1807-9288. 
di posta, alle banche online o a eBay! E una memoria cifrata ci lega ai nostri soldi, ogni volta che usiamo un bancomat e ci viene richiesto il PIN. Quanti espressioni dei nostri corpi riproduciamo attraverso le emoticone!

Le emoticone, o icone emozionali, sono il genere di segni scritti al momento tra i più universalmente e comunemente utilizzati. II termine icona in semiotica indica un particolare tipo di "segno" che è in una relazione di somiglianza con ciò che rappresenta. Basate sulla combinazione tra i segni di interpunzione, le emoticone hanno la caratteristica principale di integrare scrittura e immagine allo scopo di sottolineare ironia, complicità, dispiacere, sberleffo. Nelle più sofisticate interfacce delle chat, nelle applicazioni per smartphone e tablet, la semplice giustapposizione dei segni di interpunzione che noi combiniamo sulla tastiera si traduce in vere e proprie faccine espressive e, volendo, anche animate, in modo tale da dare alla scrittura una "vitalità" visiva che combina lettere e disegni, come avviene nei rebus. Anche nella loro forma più elementare, comunque, tali segni evocano fondamentalmente due aspetti: la dimensione costitutivamente iconica del linguaggio e la presenza della corporeità nella parola. Se questa dimensione trova nel linguaggio orale la sua naturale estrinsecazione attraverso quelli che si chiamano aspetti paralinguistici e tratti sovrasegmentali del linguaggio, come $i$ gesti delle mani e della bocca, l'espressione del volto, il rossore, la posizione del corpo, nel testo scritto in prosa, prima dell'invenzione delle emoticone, essi non avevano modo di venire espressi se non attraverso parentesi, punti esclamativi, note esplicative. Le emoticone riescono invece a concentrare in pochi tratti gli aspetti del linguaggio che si collegano alla dimensione corporea, emozionale, perfino passionale. Nella chat le emoticone sopperiscono per esempio alla riduzione di efficacia del principio fondamentale della conversazione, che è il principio di cooperazione tra parlanti e che si esprime al massimo livello quando la conversazione avviene in presenza. La gestualità, l'intonazione, il ritmo, la velocità dell'eloquio possono così rendersi trasparenti anche nella scrittura. II corpo, per meglio dire il volto, ridotto a uno schizzo tracciato in pochi tratti, viene rappresentato in modo buffonesco, ironico, parodico. In questo senso, si realizza quella pratica linguistica che potremmo chiamare con un neologismo che unisce sincreticamente in sé oralità e scrittura: l'oralitura.

Texto Digital, Florianópolis, Santa Catarina, Brasil, v. 11, n. 2, p. 148-170, jul./dez. 2015. ISSNe: 1807-9288. 
Nella lingua scritta, i segni di punteggiatura e le faccine, un po' eredi dei tratti soprasegmentali, possono porre allora potenzialmente qualunque testo in grado di esprimere ciò che solo il testo poetico, per sua naturale vocazione, aveva potuto realizzare attraverso il ritmo, la metrica, il verso. Pur legato a una "macchina", il testo elettronico contiene in sé la corporeità ed esplicita il genere di chi scrive, compresa anche la possibilità di barare e di operare una detronizzazione parodica del corpo.

Altri elementi legati alla corporeità sono all'opera in quei luoghi del web, i social network, in cui si coniugano tra loro comunicazione, dimensione ludica, dimensione pratica, cultura visuale e interazione sociale. Twitter e Facebook, quelli attualmente più universalmente noti e frequentati, possono essere interpretati scorgendo in loro alcuni tratti caratterizzanti che richiamano modelli linguistici. In Facebook prevale una delle più interessanti funzioni comunicative individuate da Roman Jakobson (JAKOBSON, 1963): la funzione fàtica, quella dedicata al semplice mantenimento del livello della "presenza" nella comunicazione, quella che, ad esempio, in una telefonata esprimiamo chiedendo "Ci sei?" e rispondendo "Ci sono, ti sento". Facebook è il social network oggi più famoso, che venne concepito nel 2004 dal suo "inventore" Zuckerberg allo scopo di mantenere il contatto con gli "amici" della rete, in origine i colleghi di università; ha poi però conosciuto una evoluzione talmente rapida che il suo numero di utenti si è moltiplicato in milioni di persone in tutto il mondo e si è allargato all'universo intero, per così dire, il gruppo di "amici” potenzialmente raggiungibili da ciascuno. In questo social network, la funzione fàtica viene rappresentata innanzitutto nell'espressione di status in cui l'utente risponde alla implicita domanda "A cosa stai pensando?". Rispondendo ad essa con una frase in cui viene indicata una situazione emotiva, una preferenza politica, o un giudizio sul mondo, si vuole segnalare né più né meno che si è presenti nella rete e che l'“amicizia" può prendere avvio. Anche nella funzione del "Mi piace", introdotta dopo un paio di anni di vita e di universale successo di Facebook, la funzione fàtica si manifesta, questa volta con la dimostrazione della partecipazione emotiva allo status che 
qualcun altro ha dichiarato, al link da lui/lei richiamato, alla nota postata, alla foto o al video pubblicati.

In Twitter prevalgono invece sia la funzione referenziale sia quella informativa, evidenti nel "tweet" il "cinguettio" contenuto in 140 caratteri, entro i quali l'emittente deve comunicare il suo messaggio. Questo social network è usato infatti spesso dai giornalisti e dagli uffici stampa per comunicare in tempo reale con le rispettive redazioni o con gli altri utenti del social network.

In Facebook sono all'opera valorizzazioni sociali importanti, come il narcisismo e la ricerca di memorie perdute (vecchie foto, vecchi compagni/e di scuola), ma anche aspetti ludici e giocosi, elementi di informazione, come quelli che si realizzano nel suggerire link ad articoli a notizie e documenti online. Non va però mai dimenticato che sui social network, e su Facebook in particolare, si concentrino interessi economici miliardari legati al marketing che di per sé questo mezzo rende possibile a costo zero: stili di vita, gusti letterari, opinioni politiche entrano in una sorta di acefala e anonima banca dati del web dalla quale traggono però vantaggio società e gruppi economici a volte non disgiunti, anzi spesso consustanziali, a gruppi di potere politico. Così il "mi piace", da semplice espressione fàtica, rischia di diventare oggetto di un sondaggio. La classifica dei libri che si porterebbero in un'isola deserta o dei dischi della vita diviene elemento che produce trend.

\section{Note conclusive}

Nella nostra epoca le forme della scrittura e della lettura sono sempre più numerose e complesse, sia nella vita quotidiana che nell'attività professionale. Fa parte della nuova competenza linguistica e culturale, della nuova alfabetizzazione segnica, riuscire a cercare la giusta via da percorrere e attraverso la quale orientarsi nel labirinto del web e dei testi elettronici. Questa nuova alfabetizzazione non deve essere solo meccanica, non dobbiamo cioè solo imparare a usare tecnicamente le macchine, i software e la navigazione ipertestuale, senza sapere cosa stiamo facendo, senza conoscere e riconoscere i meccanismi semiotici, 
ideologici, sinestetici, che sono all'opera, altrimenti non facciamo altro che annegare nell'alienazione segnica.

\section{METAMORFOSE DA ESCRITURA}

Resumo: Este artigo se concentra na metamorfose que a escritura está vivendo em nosso tempo. Explica conceitos como a criatividade, a escritura, a leitura e a nominação - que mudaram na era digital - e como as TIC estão mostrando a complexidade da escritura. Para isso, recorre à "Lições americanas" (1985), do escritor italiano Italo Calvino. Apesar de ter morrido em 1985, por conseguinte, um pouco antes da revolução digital do fim do século XX, compreendeu bem os valores e as regras que a escritura teria de preservar no futuro. Suas "recomendações" em matéria de leveza, rapidez, exatidão, visibilidade e multiplicidade: palavras-chave que eu comparo com a sua eventual aplicação na era das conexões planetárias.

Palavras-chave: Escritura. Leitura. Idioma. TIC. Metamorphosis.

\section{METAMORPHOSIS OF WRITING}

ABSTRACT: This article focuses on the metamorphosis that writing is living in our age. I would explain how concepts like creativity, writing, reading, naming, have changed in the digital age and how ICTs are showing the complexity of writing. To do that, I would follow the ideal "guide" of the "American lectures" Six Memos for the Next Millenium (1985), by the Italian writer Italo Calvino. Although he died in 1985, Calvino grasped very well both the values and the rules that writing should have preserved in the future. His Memos concern lightness, quickness, exactitude, visibility and multiplicity: key words that I will compare with their possible fulfillment in the age of planetary connections.

KEYWORDS: Writing. Reading. Language. ICTs. Metamorphosis.

\section{Riferimenti}

BARTHES, Roland. Saggi critici. Torino: Einaudi, 2002.

CALVINO, Italo. 1985. Lezioni americane. Saggi. 1945-1985. Milano: Mondadori 1995. 
CARDONA, Giorgio Raimondo. Antropologia della scrittura. Torino: Loescher, 1981.

DE MAURO, Tullio. Capire le parole. Roma-Bari: Laterza, 1999.

DERRIDA Jacques. Della grammatologia. Milano: Jaca Book, 1988.

— Eperons. Les styles de Nietzsche. ed. in 4 lingue, Venezia: Corbo \& Fiore, 1976

JAKOBSON, Roman. Saggi di linguistica generale. Milano: Feltrinelli, 1972.

NEGROPONTE, Nicholas. Essere digitali. Milano: Sperling \& Kupfer, 1995.

Platone. Fedro, trad. it. di P. Pucci. Opere complete. Roma-Bari: Laterza, 1974, vol. 3.

PONZIO, Augusto. "Dare voce a Barthes". Con Roland Barthes, alle sorgenti del senso, a c. di A. Ponzio, P. Calefato, S. Petrilli, Roma: Meltemi, 2006, p. 15-43.

ROSSI-LANDI, Ferruccio. Significato, comunicazione e parlare comune. Venezia: Marsilio, 1980.

SANGA, Glauco. "Antropologia della scrittura: origini e forme". Scrittura e scritture: le figure della lingua. Atti del XXIX Convegno della Società italiana di Glottologia, a c. di M. Mancini e B. Turchetta, Roma: II Calamo di Fausto Liberati snc, 2009, pp. 13-66.

Texto recebido em: 19/10/2015.

Texto aceito em: 11/12/2015. 